\title{
Diskurs hin zu einer unterrichtszentrierten Schulentwicklung in Richtung personalisierten Lernens
}

Der Diskurs hin zu einer Schul- und Unterrichtsentwicklung in Richtung personalisierten Lernens wird im Folgenden entlang von zwei Diskussionssträngen beschrieben: Erstens wird der deutschsprachige Schulentwicklungsdiskurs dargestellt, in dem Prozessgestaltung, Potenziale und Herausforderungen in der Weiterentwicklung von Einzelschulen erörtert werden (Abschnitt 2.1) und zunehmend die Unterrichtsentwicklung ins Zentrum gestellt wird (Abschnitt 2.2). Um den Bereich der Unterrichtsentwicklung näher beleuchten zu können und Merkmale didaktischen Handelns für verstehensbezogene und nachhaltige Lernprozesse eruieren zu können, orientiert sich diese Arbeit zweitens am Diskurs der pädagogisch-psychologischen Lehr- und Lernforschung. Insbesondere die Frage nach einer stärker personalisierten Gestaltung von Lehr- und Lernprozessen steht hier im Fokus (Abschnitt 2.3).

\subsection{Schulentwicklung als Entwicklung von Einzelschulen}

Seit es öffentliche Schulen als institutionelle Einrichtung für Bildung und Erziehung gibt - in Zürich wurde 1832 das erste Schulgesetz demokratisch verabschiedet (Oelkers, 2015) - fanden immer wieder Phasen von Entwicklung statt. Beispielsweise wurden Lehr- und Bildungspläne in den ersten dreißig Jahren des 20. Jahrhunderts umfassend erweitert und stärker an die kognitive Entwicklung und die Persönlichkeitsentwicklung der Schülerinnen und Schüler angepasst, wodurch erstmalig die Lernmöglichkeiten und die Lerninteressen von Kindern und Jugendlichen mitberücksichtigt wurden (Fend, 2006). Die Weiterentwicklung solcher Bemühungen bis in die heutige Zeit wird nachfolgend kurz nachgezeichnet. 
Schulentwicklung als äußere Schulentwicklungsplanung

Der Begriff „Schulentwicklung“ etablierte sich im deutschsprachigen Raum erst in den 1970er-Jahren. Maßgebenden Anteil daran hatten die folgenden beiden, Anfang der 1970er-Jahre gegründeten Institutionen: das Zentrum für Schulversuche und Schulentwicklung in Österreich und das Institut für Schulentwicklung in Deutschland (Altrichter \& Helm, 2011; Rolff, 2007). Unter „Schulentwicklung“ war das Vorgehen zu verstehen, dass Akteurinnen und Akteure der Systemebene, vor allem Personen mit Entscheidungsbefugnis aus der Bildungspolitik und der Schulbehörde, durch gezielte Maßnahmen Reformimpulse setzten, die von den Schulen aufgenommen und zur intendierten Veränderung führen sollten. Entwicklung wurde damals verstanden als strukturelle Schulentwicklungsplanung, in der auf äußere Schulangelegenheiten wie Standort, Raumkapazität, Gebäude etc. Einfluss genommen wurde (Rolff, 1993, 2007).

\section{Schulentwicklung als Entwicklung von Einzelschulen}

In den 1980er- und 1990er-Jahren zeigten Studien (Dalin, 1971; Fend, 1982; Fullan, 1991; Huberman \& Miles, 1984), dass schulexterne, top-down durchgeführte Schulentwicklung die angestrebten Ziele nicht erreicht, weil jede Schule die Ziele und Vorgaben anhand der kontextspezifischen Rahmenbedingungen adaptiert. Mit anderen Worten hängt der Erfolg einer Schulreform von konkreten Umsetzungsmöglichkeiten und vom Handeln der Schulleitenden und der Lehrpersonen selbst ab (u. a. Altrichter \& Helm, 2011). Einzelschulen wurden vermehrt als pädagogische Handlungseinheiten verstanden, die ihren Gestaltungs- und Autonomieraum eigenständig bearbeiteten (Fend, 1986, 2017). In diesem Zeitraum wurde in der Schweiz die Funktion der Schulleitenden eingeführt, die je nach Kanton unterschiedliche Verantwortungs- und Aufgabenbereiche haben. Schulleitende verfügen über Handlungskompetenzen, die es ihnen ermöglichen, Prozesse der Schul- und Unterrichtsentwicklung selbst zu gestalten (Trachsler, 2012). Dies bildet eine wichtige Grundlage für die Implementierung neuer Unterrichtskonzepte, zum Beispiel von Konzepten personalisierten Lernens.

\section{Entwicklung der Einzelschule, flankiert von neuen Steuerungsinstrumenten}

Seit Anfang der 2000er-Jahre werden Ergebnisse internationaler Vergleichsstudien (large-scale studies; z. B. TIMSS, PISA) intensiv diskutiert. Die Ergebnisse von Leistungstests und die daraus resultierenden Rangreihen der teilnehmenden Länder lösten besonders in Deutschland intensive Debatten u. a. über die Leistungsfähigkeit des Bildungssystems aus (Stichwort „PISA-Schock“, u. a. Hildebrandt, 2009; Schwippert \& Goy, 2008). Seither werden vermehrt Steuerungsinstrumente 
wie beispielsweise externe Schulevaluationen, standardisierte Lernstandserhebungen und Schulinspektionen eingesetzt, die u. a. darauf abzielen, Schul- und Unterrichtsentwicklung an Einzelschulen zu initiieren und qualitätsbezogen abzusichern (Altrichter \& Helm, 2011; Bohl, 2009; Kohler \& Wacker, 2013; Terhart, 2000). Mit den neuen Steuerungsinstrumenten geht die Forderung nach einer Rechenschaftspflicht (accountability) der Einzelschulen einher: Schulleitende und Lehrpersonen sollen einerseits ihre geleistete (Entwicklungs-)Arbeit transparent darlegen und andererseits durch zurückgemeldete Evaluationsergebnisse weitere Entwicklungsmöglichkeiten erhalten (Altrichter \& Maag Merki, 2016a). Damit wird ein verwaltungstechnisches Top-down-Verständnis von Schulentwicklung erweitert durch ein Verständnis, nach dem mittels auf der Basis von Evaluationen gesetzten Gestaltungsimpulsen die Schulleitenden und die Lehrpersonen gemeinsam mit weiteren Akteursgruppen auf der Systemebene die Entwicklung des Bildungssystems kooperativ vorantreiben (Altrichter \& Maag Merki, 2016b; Fend, 2011). Die in den meisten Kantonen der Schweiz eingesetzten externen Schulevaluationen oder die seit 2016 erstmals landesweit durchgeführten standardisierten Tests zur Überprüfung der Erreichung von Grundkompetenzen (ÜGK ${ }^{1}$ ) sind Beispiele dieser Entwicklung. Solche neuen Steuerungsinstrumente bilden auch in Schulen, in denen Lehr- und Lernprozesse vermehrt personalisiert gestaltet werden, einen Rahmen für Prozesse der Schul- und Unterrichtsentwicklung.

\subsection{Unterrichtsentwicklung im Zentrum von Schulentwicklung}

Mit den neuen Steuerungsinstrumenten sollen besonders Veränderungen auf der Unterrichtsebene initiiert werden. Das ,pädagogisch-professionelle Handeln und [...] die Effektivität [von] Innovationen für das Lernen der Schüler/innen“ (Emmerich \& Maag Merki, 2014, S. 6) stehen im Zentrum und Schulentwicklungsmaßnahmen sollen Lernumgebungen dahingehend verbessern, dass die Schülerinnen und Schüler nachhaltiger lernen und höhere Lernerträge erzielen können (Maag Merki, 2018). Dieser Fokus auf den Unterricht wird im Folgenden kurz erläutert.

\section{Gründe der Unterrichtszentrierung}

Der Gedanke, Schulentwicklung ganz zentral als Unterrichtszentwicklung zu verstehen, hat seit den 2000er-Jahren stetig an Bedeutung gewonnen. Die Gründe

\footnotetext{
${ }^{1}$ Quelle: www.uegk-schweiz.ch [letzter Zugriff: 01.01.2021].
} 
hierfür sind vielfältig. So erfordern zum Beispiel veränderte Rahmenbedingungen des Unterrichts (heterogene Lerngruppen, kompetenzorientierter Unterricht etc.) nicht mehr nur einen eng geführten Klassenunterricht, sondern vielfältige Lehr- und Lernformen, in denen die Schülerinnen und Schüler kooperativ und selbstständig lernen können und in denen durch adaptive Lernunterstützung und kognitiv anregende, problembasierte Aufgabenstellungen verstehensbezogene Lernprozesse ausgelöst werden (Reusser et al., 2013; Stebler et al., 2018). Hierfür sind Entwicklungstätigkeiten nötig, die über die Entwicklung und die Steuerung der Einzelschule als Organisationseinheit hinausgehen (u. a. Schlee, 2014). Zu diesem Zweck werden Ansätze der Organisationsentwicklung, wie etwa der ISP von Dalin und Rolff (1990), mit einer Unterrichtsperspektive erweitert. Besonders Erkenntnisse aus der pädagogisch-psychologischen Lehr- und Lernforschung treiben den Diskurs über optimale Lernumgebungen für jede Schülerin und jeden Schüler an. Erwähnenswert sind hier Videostudien, in denen konkrete Handlungen beobachtet und analysiert werden, um daraus Merkmale qualitätsvoller Lehr- und Lernarrangements ableiten zu können. Auf diese Weise wird es möglich, Mikroprozesse im Unterricht zu untersuchen, welche die psychologischen Tiefenschichten des Lernens der Schülerinnen und Schüler positiv beeinflussen (Bonsen, 2010; Faust, Lipowsky \& Kastens, 2013; Reusser, 2017; Reusser et al., 2010). Eine weitere diesbezüglich relevante Forschungsarbeit hat der Neuseeländer Hattie $(2009,2012)$ vorgelegt. Mit seiner Analyse von mehr als 900 Metaanalysen untersuchte er, was im Unterricht effektiv wirkt (,What works best?“). Seine Ranglisten von Faktoren und deren Effektstärken zeigen deutlich, dass die Lehrpersonen mit einem qualitätsvollen Lehrhandeln eine wichtige Rolle für den Lernerfolg der Schülerinnen und Schüler spielen. Durch das aufwendige Design und die Art und Weise, in der Hattie die Ergebnisse der wissenschaftlichen Gemeinschaft präsentiert, sowie angesichts des breiten Interesses der Öffentlichkeit an der Studie, ${ }^{2}$ nicht zuletzt infolge der Übersetzung in die deutsche Sprache durch Beywl und Zierer, kann dieser Meta-Metaanalyse durchaus ein Einfluss auf den deutschsprachigen Schulentwicklungsdiskurs zugesprochen werden.

\section{Beispiele von Konzepten einer unterrichtszentrierten Schulentwicklung}

Einen im Diskurs zu unterrichtszentrierter Schulentwicklung kaum beachteten Ansatz stellte Wolfgang Klafki bereits 1985 vor. In seinem Aufsatz über die innere

\footnotetext{
${ }^{2}$ Zum Beispiel der Beitrag aus der Aargauer Zeitung: www.aargauerzeitung.ch/leben/wasist-guter-unterricht-star-bildungsforscher-john-hattie-erklaert-wie-kinder-am-besten-lernen132486887 [letzter Zugriff: 01.01.2021].
} 
Schulreform beschreibt er die didaktische Unterrichtsgestaltung und deren Entwicklung als deren zentrales Element. Die innere Schulreform umfasst das Schulleben, die Schulatmosphäre sowie Ziele, Inhalte und Methoden des Unterrichts, während sich die äußere Schulreform auf schulorganisatorische Aspekte bezieht. Klafki erläutert seinen Ansatz am Beispiel der Gesamtschule und thematisiert nicht nur Unterrichtsmethoden wie etwa Projektunterricht oder fächerübergreifenden Unterricht, sondern auch didaktische Konzepte wie soziales Lernen, innere Differenzierung oder die individuelle Förderung von Schülerinnen und Schülern in heterogenen Lerngruppen (Klafki, 1985/2007).

Ein weiteres Beispiel ist das bereits in der Einleitung (siehe Abschnitt 1.1) erwähnte Schulentwicklungsprojekt „Erweiterte Lernformen“ (ELF) der Nordwestschweizerischen Erziehungsdirektorenkonferenz (NW EDK): Zwischen 1990 und 1995 wurden in sieben Kantonen und in 85 Teilprojekten mit ca. 1200 Lehrpersonen offenere, den lehrpersonenzentrierten Klassenunterricht ergänzende Unterrichtsformen erarbeitet und eingesetzt, die Elemente der inneren Differenzierung und der Individualisierung enthielten. In den entsprechenden Lernumgebungen konnten die Schülerinnen und Schüler zu einem gewissen Grad selbst über ihre Lernprozesse entscheiden und gemeinsam mit anderen Schülerinnen und Schülern Lernaufgaben kooperativ lösen (u. a. Croci et al., 1995; Landwehr, 1996). Beispiele solcher erweiterten Lehr- und Lernformen sind Werkstattunterricht, Wochenplan oder Projektunterricht. Die konzeptionelle Anlage des Projekts war in zweifacher Hinsicht besonders. Erstens zielten die Entwicklungsimpulse unter Berücksichtigung lernpsychologischer Erkenntnisse auf die didaktische Gestaltung des Unterrichts ab (Croci et al., 1995): Eigenständig bearbeitete Problemstellungen und die Reflexion der eigenen Lernprozesse sollten zu verstehensbezogenem Lernen anregen. Dabei wurden Lehrpersonen im Vergleich mit eng geführtem Klassenunterricht weniger als Instruktorinnen und Instruktoren oder reine Wissensvermittelnde angesehen, sondern vielmehr als Lernbegleitende und Lernunterstützende (u. a. Pauli et al., 2003). Zweitens war in diesem Projekt die Unterrichtsentwicklung zentral, die von Entwicklungstätigkeiten auf Schulebene umrahmt werden sollte: Lehrpersonen wurden dazu angeregt, u. a. in internen Weiterbildungen gemeinsam erweiterte Lehr- und Lernformen zu erarbeiten und diese im eigenen Unterricht zu erproben, während die beteiligten Schulen angehalten wurden, ein „nach aussen wahrnehmbares Profil [zu] entwickeln“ (Croci et al., 1995, S. 7).

Im Zuge der Umsetzung der UN-Behindertenrechtskonvention von 2006 und zunehmend heterogener werdender Lerngruppen werden vermehrt Konzepte inklusiver Schulentwicklung erarbeitet (u. a. Moser \& Egger, 2017). Darin steht die Entwicklung von Lernumgebungen im Zentrum, an denen alle Schülerinnen 
und Schüler mit unterschiedlichen Lernvoraussetzungen teilhaben können, bei gleichzeitiger Minimierung von Benachteiligung und Diskriminierung (Werning, 2011). Im Zusammenhang mit der Entwicklung solcher Lernumgebungen werden neben der Unterrichtsentwicklung noch weitere Tätigkeitsfelder beschrieben, bei Lozano, Vetterli und Wyss (2017) etwa die Kooperation der Lehrpersonen, das Mitwirken der Eltern oder auch die Einstellungen und Haltungen der Lehrpersonen gegenüber der Integrations-/Inklusionsidee. Alle Tätigkeitsfelder beeinflussen sich wechselseitig und verfolgen das Ziel, inklusiven Unterricht zu ermöglichen.

Im Ansatz einer pädagogischen Schulentwicklung nach Klippert (2008) bilden heterogene Lerngruppen einen Ausgangspunkt für Weiterentwicklung. Auch hier stehen Lehr- und Lernformen im Zentrum, in denen die Schülerinnen und Schüler Lerninhalte in individuellen oder kooperativen Lernsettings selbstständig bearbeiten können. Klippert knüpft u. a. an Erkenntnissen der Lernpsychologie an, die aufzeigen, dass Lernprozesse dann nachhaltig sind, wenn sie aktiv und verstehensbezogen gestaltet sind. Seine ausführliche Beschreibung des Methodentrainings für Lehrpersonen wird gerahmt von der Beschreibung weiterer Tätigkeiten auf Schulebene: netzwerkartige Kooperation zwischen Lehrpersonen und Schulleitenden, Zusammenarbeit mit den Eltern, Einbezug von Beratenden sowie ein systematisches Innovationsmanagement.

Die Ausführungen von Bastian (2007) zur unterrichtszentrierten Schulentwicklung zielen weniger auf ein Methodentraining ab wie bei Klippert (2008), sondern vielmehr auf eine fundierte theoretische Einbettung von Unterrichts- und Schulentwicklung mit Bezügen zur Unterrichtsqualität, zur Professionalisierung von Lehrpersonen sowie zu Selbstregulation und Feedback im Lernprozess. Bastian setzt diese Tätigkeiten auf Unterrichtsebene in Bezug zur Schul- und Systemebene: Ausgehend von der Entwicklung des Unterrichts sollen die Tätigkeiten in die gesamte Schule sowie in die regionale Bildungslandschaft eingebunden werden. Allerdings nennt er weniger Entwicklungstätigkeiten beteiligter Personen (z. B. von Lehrpersonen und Schulleitenden) auf Schulebene und beschreibt das Zusammenwirken von Unterrichts- und Schulentwicklung weniger systematisch als beispielsweise im oben aufgeführten Konzept inklusiver Schulentwicklung von Lozano et al. (2017). 


\subsection{Unterrichts- und Schulentwicklung in Richtung personalisierten Lernens}

Die im Rahmen des Projekts „Erweiterte Lernformen“ der NW EDK initiierte Weiterentwicklung der didaktischen Unterrichtsgestaltung hin zu differenzierenden und individualisierenden Lernumgebungen und die Förderung unterrichtszentrierter Kooperation zwischen Lehrpersonen sind zwei zentrale Elemente einer unterrichtszentrierten Schulentwicklung in Richtung personalisierten Lernens. Somit haben das Projekt der NW EDK sowie weitere Entwicklungsinitiativen rund um die erweiterten Lehr- und Lernformen wichtige Vorarbeiten für heutige Unterrichtsentwicklung in Schweizer Volksschulen geleistet, die nachfolgend mit Fokus auf den Aspekt der Personalisierung im Überblick dargestellt wird.

\section{Unterrichtsentwicklung in reformpädagogischen Schulen}

Ansätze des geöffneten Unterrichts sind in - meist privaten - Reformschulen schon viel länger etabliert als in der Volksschule. $\mathrm{Zu}$ nennen sind diesbezüglich zum Beispiel Johann Heinrich Pestalozzi (1746-1827) mit dem Institut in Burgdorf und später in Yverdon sowie Philipp Emanuel von Fellenberg (1771-1844) mit dem Schulstaat in Hofwyl bei Bern. Beide Einrichtungen verfügten über ein Unterrichtskonzept, das die Lernbedürfnisse der Kinder besonders berücksichtigte, wobei die Umsetzungsqualität in pädagogischer und didaktischer Hinsicht insbesondere im ersteren Fall jedoch kritisch zu beurteilen ist (Oelkers, 2015; Osterwalder \& Reusser, 1997). ${ }^{3}$

In der angloamerikanischen Literatur zu personalized learning werden oft die Arbeiten von John Dewey (1859-1952) rezipiert (u. a. Bray \& McClaskey, 2015; Mincu, 2012a; Yonezawa, McClure \& Jones, 2012; Zmuda, Curtis \& Ullman, 2015). In seinem Unterrichtskonzept gestalten Lehrpersonen Lernumgebungen ausgehend von den individuellen Fähigkeiten, Fertigkeiten, Interessen und weiteren Lernbedürfnissen der Kinder. Zentral sind Problemstellungen als Lerngegenstand. Alltagsnahe und kindgerechte Problemstellungen sollen die Schülerinnen und Schüler kognitiv herausfordern und von diesen selbstständig sowie kooperativ bearbeitet werden (Dewey, 1910/2002; Schönig, 2018). Ein weiteres im angloamerikanischen Sprachraum bekanntes reformpädagogisches Konzepte ist neben dem Winnetka-Plan von Washburne (1927), bei dem u. a. die Schülerinnen und Schüler in altersdurchmischte Leistungsgruppen aufgeteilt wurden und neben dem fachlichen Lernen auch das soziale Lernen als Mitglied

\footnotetext{
${ }^{3}$ Eine kritische Auseinandersetzung mit reformpädagogischen Ansätzen kann u. a. bei Oelkers (2005) nachgelesen werden.
} 
einer Gemeinschaft einen zentralen Aspekt darstellte, der Dalton-Plan von Parkhurst (1922). Parkhurst ging, wie viele der reformpädagogischen Ansätze, von einer Kritik des in der damaligen Zeit etablierten Schulsystems aus. Insbesondere kritisierte sie die direktiven und frontalen Unterrichtsformen, welche der individuellen Entwicklung des Kindes wenig Raum lassen würden. Daraus leitete sie das zentrale Ziel von Unterrichten ab: ,The true business of school is not to chain the pupil to preconceived ideas, but to set him free to discover his own ideas and to help him to bring all his power to bear upon the problem of learning" (Parkhurst, 1922, S. 151 f.). Die Autorin setzte das Lernen der Schülerinnen und Schüler ins Zentrum und ließ diese ihr eigenes Curriculum anhand ihres Könnens, ihrer Möglichkeiten und ihrer Interessen selbst gestalten. Darüber hinaus sollte nicht nur die individuelle Unabhängigkeit gefördert werden, sondern auch die soziale Eingebundenheit in einer Gruppe und die daraus hervorgehende Verantwortung für die Gemeinschaft. Ein gemäß Mincu (2012b) historisch ebenfalls wichtiger Wegbereiter personalisierten Lernens ist einer der akademischen Lehrer Deweys: Granville Stanley Hall. Er war Nestor der Child Study Movement, der frühen Kinder- und Entwicklungspsychologie in den USA, und forschte zur kindlichen Entwicklung in der Entscheidungsfindung. Die Befunde bestärkten Reformpädagoginnen und Reformpädagogen in ihren Entwicklungs- und Lernvorstellungen, dass das Kind natürlicherweise wissbegierig ist und eigenaktive Lernen kann (Konrad, 2017).

\section{Anfünge von Unterrichtsentwicklung unter der Leitidee personalisierten Lernens}

Die Ausführungen zur Unterrichtsentwicklung in reformpädagogischen Schulen zeigen auf, dass die Idee von individualisierten und differenzierenden Lernumgebungen, in denen die Schülerinnen und Schüler über vergrößerte Autonomie- und Handlungsspielräume bezüglich ihrer Lernprozesse verfügen, nicht neu ist. Neu ist dagegen, dass diese Idee unter dem Terminus personalized learning diskutiert wird und auch in Volksschulen zunehmend Einzug hält. Erste Nennungen von personalized learning lassen sich vor der Jahrtausendwende in den USA finden. Zu den ältesten Publikationen, die sich in einer diesbezüglichen Literaturrecherche finden lassen, zählt u. a. diejenige von Spaulding (1970), der eine Intervention für benachteiligte Schülerinnen und Schüler beschreibt, in deren Rahmen diese in ihrem Lernen mehr Freiheiten erhielten und Verantwortung für ihre Lernentscheidungen übernahmen. LaJeunesse und Hansen (1966) wiederum stellten ein Personalized Curriculum Program vor, mit dessen Hilfe durch individuelle Lernprogramme, persönliche Lernunterstützung sowie eine stärkere Orientierung an der Berufswelt eine bestmögliche Integration benachteiligter Schülerinnen und Schüler in die Gesellschaft erreicht werden sollte. Als drittes Beispiel zu nennen ist schließlich Peck (1970), der zum Programm Personalized Education im 
US-Bundesstaat Texas Stellung nahm. Gemeinsam haben die drei ,historische“ Beispiele, dass personalized learning als didaktisches Unterrichtsmodell damals erst mit wenigen Bezügen zur Schulebene konzipiert wurde und diese Bezüge eher impliziter ausfielen (Jenkins \& Keefe, 2002; Watkins, 2012).

Personalisiertes Lernen im Kontext von Schulentwicklung und Bildungsreformen im englischsprachigen Bildungsraum

Der Blick über den Unterricht hinaus auf die Schulebene und auf dieser Ebene notwendige Veränderungen erfolgte erst in Publikationen, die seit den 2000erJahren erschienen sind. Es wird eruiert, welche Bedingungen und Tätigkeiten erforderlich sind, damit Konzepte des personalisierten Lernens nicht nur bei einzelnen Lehrpersonen nachhaltig implementiert werden, sondern in der gesamten Einzelschule. Beispiele hierfür sind Arbeiten von D. Hargreaves (2006a, 2006b), Bray und McClaskey (2015) oder Prain et al. (2015c). Aber auch in den politischen Parteiprogrammen und Bildungsreformen ab den 1990er-Jahren, bei denen in Ländern wie den USA, Großbritannien oder Australien Konzepte personalisierten Lernens in Bildungsregionen eingeführt werden sollten, sind Beispiele zu finden (Keamy, Nicholas, Mahar \& Herrick, 2007; Watkins, 2012). So wurde etwa in Australien in der Adelaide Declaration on National Goals for Schooling in the Twenty-first Century (Ministerial Council on Education, 1999) Schlüsselelemente personalisiertes Lernen aufgeführt. Hierunter wurden lebenslanges Lernen, die Schule als lernende Gemeinschaft und der Gebrauch von ICT gefasst. Im Jahr 2007 wurde personalisiertes Lernen im Bericht The Future of Schooling in Australia (Council for the Australian Federation, 2007) - neben qualitätsvollem Unterrichten und Engagement der Schule in der Gemeinschaft - als zentrales Element deklariert (Keamy et al., 2007). In Großbritannien war personalisiertes Lernen während der Regierungszeit von Tony Blair ein bildungspolitisches Reformprogramm. In Every Child Matters (Department for Education and Skills, 2004a) sowie New Relationship with Schools (Department for Education and Skills, 2004c) wurden Formen personalisierten Lernens als geeignet beschrieben, um die angestrebten Bildungsziele zu erreichen (Deakin-Crick, 2012). David Miliband, damaliger britischer Staatsminister, stellte 2004 den Konzeptrahmen unter dem Titel Choice and Voice in Personalised Learning - The Future of Public School Reform mit den folgenden sechs Eckpunkten vor (Miliband, 2006):

- assessment for learning,

- effective teaching and learning,

- curriculum entitlement and choice,

- a student-centered approach to school organisation und 
- strong partnership beyond the school.

In den darauffolgenden Jahren finden sich Hinweise in anderen Ländern, etwa Neuseeland oder Kanada, in denen personalisiertes Lernen im bildungspolitischen Kontext diskutiert wurde und nach wie vor diskutiert wird (Stebler et al., 2018). Zum heutigen Zeitpunkt lässt sich ganz generell festhalten, dass die Auseinandersetzung mit personalisierten Formen der Unterrichtsgestaltung mittlerweile weltweit stattfindet: „It is a new global education policy about the personalisation of learning and curricula“ (Beach \& Dovemark, 2009, S. 690).

\section{Personalisiertes Lernen im deutschsprachigen Bildungsraum}

Im deutschsprachigen Bildungsraum ist der Begriff „personalisiertes Lernen“ wenig verbreitet. Mit der Sucheingabe ,personali* AND lern*“ lässt sich im Fachportal Pädagogik als älteste Publikation diejenige von Niehaus (2003) finden. Er beschreibt eine internetbasierte Wissensorganisation in der Lehrpersonenausbildung, die den Nutzenden mittels Online-Werkzeugen personalisierte Lerninhalte zur Verfügung stellt. Die Idee, mittels (Lern-)Programmen einen an die Nutzenden angepassten Lernweg anzubieten, wird in der ICT-Branche bereits seit vielen Jahren verfolgt (u. a. Keller, 1968). Solche Lernprogramme sammeln anhand der Zeit und der Richtigkeit von gelösten Aufgaben Informationen, auf deren Grundlage sie angepasste Folgeaufgaben generieren (Petko et al., 2017).

Eine Publikation mit ähnlicher Datierung, die jedoch einen anderen Zugang und einen anderen Kontext aufweist, stammt von Weigand (2004). Weigand erörtert auf der Basis eines geisteswissenschaftlichhermeneutischen Ansatzes ein umfassendes anthropologisches Konzept einer personalen Schule, in der das auf eigenen Interessen und Bedürfnissen beruhende Lernen von Schülerinnen und Schülern im Zentrum steht. Ein weiterer zentraler Punkt der ,personalen Schule“ ist das Wechselspiel zwischen individuellem Lernen und gemeinschaftlichem Lernen. Diesen Ansatz haben einige Autorinnen und Autoren aufgegriffen und die Arbeit weiter vorangetrieben. Zu nennen sind hier Aufsätze von Hackl (2011, 2014) sowie Hübner und Weiss (2017) oder das Buch von Hackl und Schmid (2006). Aufbauend auf diesen Arbeiten bieten die beiden letztgenannten Autoren in Deutschland eine Weiterbildung an, welcher personorientierte Pädagogik zugrunde liegt. ${ }^{4}$ Auch in der Schweiz werden Weiterbildungen zu personalisiertem Lernen angeboten (schulentwicklung.ch). Seminarleiter war der ehemalige

\footnotetext{
${ }^{4}$ https://www.ewib.de/ [letzter Zugriff: 01.01.2021].
} 
Schulleiter des Instituts Beatenberg, Andreas Müller. Er war zudem Autor zahlreicher Texte und Bücher, in denen er das Konzept des personalisierten Lernens am Institut Beatenberg erläuterte (Müller, 2014, 2015).

Bei Publikationen aus der empirischen Bildungsforschung lassen sich mit der Suche nach dem Stichwort ,personalisiertes Lernen“ insbesondere diejenigen des perLen-Projekts finden (u. a. Pauli, Reusser \& Stebler, 2018; Reusser, Pauli \& Stebler, 2015b; Stebler et al., 2017, 2018). Zudem weisen die Studie zu Schulen im alpinen Raum (u. a. Raggl, Smit \& Kerle, 2015) und die Begleitforschung zu Gemeinschaftsschulen in Baden-Württemberg (u. a. Bohl \& Wacker, 2016) viele Überschneidungspunkte mit der in der vorliegenden Arbeit verwendeten Begriffsbestimmung personalisierten Lernens auf. Im Kontext der Forschung in Baden-Württemberg erschien auch ein Praxisbuch mit dem Titel „Schulen auf dem Weg zu personalisierten Lernumgebungen“ (Zylka, 2017). Ein weiterer Praxisleitfaden thematisiert die Möglichkeiten personalisierten Lernens beim Einsatz digitaler Medien (Holmes, Anastopoulou, Schaumburg \& Mavrikis, 2018). Abschließend sind die Arbeiten von Schratz (2009) sowie Schratz und WestfallGreiter (2010) zu nennen, die zwischen „lehrseits“, das heißt einem von der Lehrperson aus gedachten Unterricht, und „lernseits“, einem von den Schülerinnen und Schülern aus gedachten Unterricht, unterscheiden. In den Ausführungen plädieren die Autorin und der Autor für eine lernseitige Lernumgebung, die viele Aspekte personalisierten Lernens umfasst. Diese werden auch in neueren Publikationen hervorgehoben, zum Beispiel derjenigen von Agostini, Schratz und Risse (2018).

Open Access Dieses Kapitel wird unter der Creative Commons Namensnennung 4.0 International Lizenz (http://creativecommons.org/licenses/by/4.0/deed.de) veröffentlicht, welche die Nutzung, Vervielfältigung, Bearbeitung, Verbreitung und Wiedergabe in jeglichem Medium und Format erlaubt, sofern Sie den/die ursprünglichen Autor(en) und die Quelle ordnungsgemäß nennen, einen Link zur Creative Commons Lizenz beifügen und angeben, ob Änderungen vorgenommen wurden.

Die in diesem Kapitel enthaltenen Bilder und sonstiges Drittmaterial unterliegen ebenfalls der genannten Creative Commons Lizenz, sofern sich aus der Abbildungslegende nichts anderes ergibt. Sofern das betreffende Material nicht unter der genannten Creative Commons Lizenz steht und die betreffende Handlung nicht nach gesetzlichen Vorschriften erlaubt ist, ist für die oben aufgeführten Weiterverwendungen des Materials die Einwilligung des jeweiligen Rechteinhabers einzuholen.

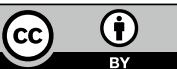

